Doi: HTTPS://DOI.ORG/10.23910/IJEP/2019.6.3.0319

\title{
Comparative Response of Some Peach [Prunus persica (L.) Batsch.] Accessions for Tree, Foliage and Floral Traits
}

\author{
Suman Bodh ${ }^{1 *}$, Dinesh Singh ${ }^{2}$, Rajesh Kumar Dogra ${ }^{1}$ and Nirmla Chauhan ${ }^{1}$
}

${ }^{1}$ Dept. of Fruit Science, Dr YS Parmar University of Horticulture and Forestry, Nauni, Solan, H.P. (173 230), India ${ }^{2}$ Dr. Y.S. Parmar University of Horticulture and Forestry, Regional Horticulture Research and Training Station, Mashobra, Shimla, H.P. (171 001) India

\section{Corresponding Author}

Suman Bodh

e-mail: sumanbodh122@gmail.com

\author{
Article History \\ Article ID: IJEP0319 \\ Received in 30 ${ }^{\text {th }}$ July, 2019 \\ Received in revised form $10^{\text {th }}$ August, 2019 \\ Accepted in final form $16^{\text {th }}$ August, 2019
}

\begin{abstract}
The present investigation entitled "Comparative Response of Some Peach [Prunus persica (L.) Batsch.] Accessions for Tree, Foliage and Floral Traits" was carried out for the evaluation of sixteen peach accessions namely Shan-i-Punjab, Kanto-5, Fertilia, Yum Yong, Flordasun, Summerglo, Suncoast, FlordaBelle, Ambri, Early Redhaven, Okubo, Early Amber, Fire Prince, Belle of Georgia, Hale Haven, Nishiki for their tree, foliage and floral characters. Flordasun recorded maximum plant height $(6.13 \mathrm{~m})$ and minimum $(2.07 \mathrm{~m})$ in Nishiki. Shedding of leaves started from $12^{\text {th }}$ November (Suncoast) to $11^{\text {th }}$ December (FlordaBelle). Time of full bloom extended from as early as (29 $9^{\text {th }}$ January) in FlordaBelle to ( $20^{\text {th }}$ March) in Fertilia and Hale Haven. Pollen viability as tested in acetocarmine and in erythrosin B ranged from $91.35 \%$ in Suncoast to $98.47 \%$ in Belle of Georgia and $58.68 \%$ in Kanto-5 to 93.90\% in FlordaBelle, respectively. In vitro pollen germination was maximum (84.33\%) in Flordasun and minimum (11.54\%) in Yum Yong under 10\% sucrose solution. Pollen germination under 15\% sucrose solution was maximum (74.22\%) in FlordaBelle and minimum (11.56\%) in Ambri.
\end{abstract}

Keywords: Accession, characterization, evaluation, flowering, peach

\section{Introduction}

Peach [Prunus persica (L.) Batsch.] is an important fruit crop of Himachal Pradesh valued for its fresh and canned fruits. The fruits of peach are attractive, delicious and highly nutritious. They are very rich source of potassium, iron, fiber, vitamin $A$, vitamin $C$ and also contains high concentrations of phytochemicals such as carotenoides, flavonols and anthocyanins (Hancock and Scorza, 2008; Byrne, 2002). In India, peach is grown on a commercial scale in mid hills of Himachal Pradesh, Jammu and Kashmir, hilly area of Uttrakhand and subtropical plains of north India (Uttar Pradesh, Punjab and Haryana) to limited extent in the hill region of eastern states.

The total area under peach cultivation in Himachal Pradesh 5076 hectares with the production of 4097 metric tonnes during 2017-2018 (Government of HP, 2019). Peaches have relatively performed well at altitude ranging between 1200-1500 m amsl. July Elberta, Redhaven and Sunhaven Suncrest and Glohaven are leading cultivars. However, peach production in recent times has seen a declining trend associated with number of factors such as diseases, overdependence on a select band of cultivars coupled with global warming. Although, peach is a temperate zone fruit, low chilling peach cultivars have been developed and found suitable for sub-tropical region (Kunden et al., 2004).

Besides, the overdominance of few peach cultivars in peach growing pockets of HP resulted in a glut in the market resulting in colossal economic losses to the farmers. This situation may only be countered by broadening the base of germplasm through introduction and evaluation of new varieties of peaches.

The development of new fruit cultivars has generally been based on genetic resources. Germplasm collection, evaluation and characterization are essential stages of breeding programs and are also the decisive factors for its adoption by the orchardists. Main germplasm collection and characterization is performed by describing phenological, pomological and morphological characteristics (Yilmaz et al., 2009).

There is a vast scope to augment peach production in temperate regions of Himachal Pradesh through varietal diversification not only to meet the demand of fresh fruit market but also to provide raw material to the agro-processing units.

Keeping in mind the above and to provide an array of 
varieties, the present study was carried out on some peach germplasm accessions available in the field gene bank of NBPGR, Regional Station, Phagli, Shimla with the objective to describe and evaluate peach germplasm for growth and floral characteristics.

\section{Materials and Methods}

The present investigation entitled 'Comparative Response of Some Peach [Prunus persica (L.) Batsch.] Accessions for Tree, Foliage and Floral Traits' was carried out in the field gene bank of National Bureau of Plant Genetics Resources, Regional Station Phagli, Shimla at an elevation of $1924 \mathrm{~m}$ amsl during the year 2017-2018. The climate of location is wet temperate. The peach germplasm accessions undertaken for studies were: Ambri, Belle of Georgia, Early Amber, Kanto-5, Yum Yong, Fertilia, Flordasun, Summerglo, Hale Haven, Suncoast, Shani-Punjab, Early Redhaven, FlordaBelle, Fire Prince, Okubo and Nishiki. Three plants of each of the accession were selected for various observations. These plants are $15-18$ years old and are grafted on wild peach rootstock.

\subsection{Tree characters}

Growth habit was categorized on the basis of the geometry of the tree crown i.e., upright, semi-upright and spreading. The height of the tree was measured with the help of calibrated staff from ground level to the tip of tallest branch of the tree and it was expressed in meters $(m)$. The spread of the tree was measured by calibrated staff in two directions i.e. NorthSouth (N-S) and East-West (E-W) and the observations were recorded in meters $(\mathrm{m})$. The trunk girth was measured at the height of $15 \mathrm{~cm}$ above from the graft union with the help of measuring tape and was expressed in centimeters $(\mathrm{cm})$.

\subsection{Foliage characters}

Thirty leaves from the middle portion of the current growth were selected during the last week of July for recording observations as suggested by Beyers (1962). The time of leaf bud burst was recorded in each accession. For this, the date on which the first bud had sprouted was taken. The length of the leaf was measured by a scale from tip of the apex to the base. The width of the leaf was measured with a scale at its broadest expand. Leaf area was worked out using the digital leaf area meter (LICOR - model $3100 \mathrm{~A}$ ). Average area of ten leaves was considered as one replication and three such replications were made.

Colour charts of the Royal Horticultural Society, London were used to determine the surface colour of the leaves. Leaf shape was observed visually following Standard Cyclopedia of Horticulture (Bailey, 1963). The leaf margin was examined for the presence or absence of serration in each accession. The hairiness of the upper and lower surfaces in mature leaves of each accession was observed. Hand lens was used for observing presence or absence of hairiness. The Petiole length of fifteen petioles was measured using hand scale and average value was worked out for three replications. Presence or absence of glands was observed visually and an average of ten leaves was considered as one replication and three such replications were made. The observations on leaf fall were recorded as the date on which there was 80-85\% leaf fall.

\subsection{Time and duration of flowering}

The time of opening of first flower was recorded on which first flower opened in each accession. Date of full bloom was recorded on the date on which about $75 \%$ flowers had opened was recorded as the date of full bloom. Date of opening of last flower was recorded on which last flower opened in each accession. The duration of flowering was recorded as number of days from the date of opening of first flower to the date of opening of last flower in each accession was determined.

\subsection{Pollen studies}

Pollen viability under acetocarmine test was recorded as pollen grains were stained in acetocarmine $(2 \%)$ and erythrosin B $(0.1 \%)$ solution. Deeply stained and normal looking pollen grains were considered as viable whereas shrivelled, lightly stained or colourless pollen grains were counted as non-viable when tested with acetocarmine. In case of erythrosin $B$ the unstained pollen grains were considered viable in this method.

The freshly dehisced pollen grains were used for in-vitro pollen germination test. Different concentration of sucrose i.e. 10 and $15 \%$ sucrose solutions were used for testing pollen germination. Pollen tube growth was assessed for each cultivar under microscope after 24 hours. The pollen grains having pollen tubes at least two times longer than pollen size were considered to be germinated and percentage of germinated pollen grains under three microscopic fields was worked out.

\section{Results and Discussion}

\subsection{Tree characters}

Flordasun recorded maximum plant height $(6.13 \mathrm{~m})$ and girth $(73.67 \mathrm{~cm})$, tree spread in N-S $(5.23 \mathrm{~m})$ and in E-W direction $(5.30 \mathrm{~m})$ was maximum in Shan-i-Punjab. While, minimum tree height $(2.07 \mathrm{~m})$ and trunk girth $(15.00 \mathrm{~cm})$ was recorded in Nishiki. The tree spread was minimum in N-S $(1.30 \mathrm{~m})$ and E-W (1.74 m) in FlordaBelle and Nishiki, respectively (Table 1). Such variation in growth characters has also been reported by previous workers (Badiyala and Lakhanpal, 1994; Singh et al., 2005; Yepthomi, 2011; Jana, 2015; Chauhan, 2016).

\subsection{Foliage characters}

Leaf characters are commonly used to distinguish and identify various fruit crop species and varieties. However, in the present study, no marked variation was observed in leaf shape, surface, margin, presence and absence of glands as well as colour (Table 2) except for some in leaf length, width, area and petiole length. Maximum leaf length $(19.23 \mathrm{~cm})$ was observed in Okubo and leaf width $(5.03 \mathrm{~cm}$ ) was found in Okubo and Nishiki whereas Fertilia was recorded to have minimum values 


\begin{tabular}{llllll}
\hline \multicolumn{6}{l}{ Table 1: Tree characters of some peach accessions } \\
\hline Accession & Growth habit & Height & Girth & \multicolumn{2}{l}{ Spread $(\mathrm{m})$} \\
\cline { 5 - 7 } & & $(\mathrm{m})$ & $(\mathrm{cm})$ & $\mathrm{N}-\mathrm{S}$ & $\mathrm{E}-\mathrm{W}$ \\
\hline Shan-i-Punjab & Spreading & 6.09 & 73.67 & 5.23 & 5.30 \\
Kanto-5 & Spreading & 3.33 & 60.67 & 2.83 & 4.07 \\
Fertilia & Semi upright & 4.32 & 41.67 & 2.87 & 3.50 \\
Yum Yong & Semi upright & 3.95 & 35.00 & 3.13 & 3.03 \\
Flordasun & Spreading & 6.13 & 60.87 & 3.83 & 3.60 \\
Summerglo & Semi upright & 4.03 & 35.00 & 2.97 & 2.93 \\
Suncoast & Spreading & 2.20 & 22.67 & 2.06 & 2.20 \\
Florda belle & Upright & 2.43 & 32.50 & 1.30 & 2.09 \\
Ambri & Semi upright & 5.57 & 64.33 & 1.83 & 2.33 \\
Early red- & Spreading & 4.06 & 18.33 & 2.22 & 2.55 \\
haven & & & & & \\
Okubo & Semi upright & 2.53 & 22.67 & 2.00 & 2.53 \\
Early amber & Spreading & 3.63 & 44.00 & 2.33 & 3.51 \\
Fire prince & Spreading & 3.50 & 31.67 & 2.67 & 2.10 \\
Belle of geor- & Semi upright & 4.07 & 19.33 & 1.39 & 7.03 \\
gia & & & & & \\
Hale haven & Semi upright & 4.40 & 36.00 & 3.16 & 2.51 \\
Nishiki & Spreading & 2.07 & 15.00 & 2.27 & 1.74 \\
Mean & & 3.89 & 38.34 & 2.63 & 3.19 \\
CD ( $p=0.05$ ) & & 0.51 & 2.69 & 0.42 & 0.43 \\
\hline & & & & &
\end{tabular}

of leaf length $(11.03 \mathrm{~cm})$ and width $(2.86 \mathrm{~cm})$. Leaf area was maximum $\left(193.43 \mathrm{~cm}^{2}\right)$ in Okubo and minimum $\left(54.83 \mathrm{~cm}^{2}\right)$ in Shan-i-Punjab. The petiole length was maximum $(1.20 \mathrm{~cm})$ in Ambri and minimum $(0.40 \mathrm{~cm})$ in Hale Haven. Time of leaf emergence was earliest ( $18^{\text {th }}$ January) in FlordaBelle and last (11 ${ }^{\text {th }}$ March) in Fertilia. Shedding of leaves started from $12^{\text {th }}$ November (Suncoast) to $11^{\text {th }}$ December (FlordaBelle). Such variation in foliage characters has also been reported by various workers (Wolfe and Strang, 2010; Byrne and Anderson, 2012; Chauhan, 2016) in the past. The difference in time of leaf emergence and leaf fall in various studies might be due to genetic constitution of the cultivars and prevailing climatic conditions in the corresponding seasons.

\subsection{Time and duration of flowering}

The flower initiation was earliest in FlordaBelle on $20^{\text {th }}$ January followed by Shan-i-Punjab on $27^{\text {th }}$ January, Flordasun on $29^{\text {th }}$ January whereas, Fertilia was the last to initiate flowering on $14^{\text {th }}$ March (Table 3). The flowering (full bloom) was earliest in the last week of January in FlordaBelle and the last was Okubo, Summerglo, Fertilia and Hale Haven in third week of March. The variation in time of flowering may be due to the differences in chilling hour requirement to break bud dormancy in various accessions. This find support from the findings of Kanwar (2002), Meena et al. (2011) and Tandon (2006). The data in Table 1 indicate that FlordaBelle was earliest $\left(10^{\text {th }}\right.$ February) in terms of date of opening of last flower followed by Shan-i-Punjab (20 th February), Flordasun $\left(25^{\text {th }}\right.$ February) and Fertilia was the last to do so on $5^{\text {th }}$ April. The longest duration of flowering was recorded in Flordasun

\begin{tabular}{|c|c|c|c|c|c|c|c|c|c|c|c|c|}
\hline \multirow{2}{*}{$\begin{array}{l}\text { Acces- } \\
\text { sion }\end{array}$} & \multirow{2}{*}{$\begin{array}{l}\text { Time of } \\
\text { leaf bud } \\
\text { burst }\end{array}$} & \multirow{2}{*}{$\begin{array}{c}\text { Leaf } \\
\text { length } \\
(\mathrm{cm})\end{array}$} & \multirow{2}{*}{$\begin{array}{c}\text { Leaf } \\
\text { width } \\
(\mathrm{cm})\end{array}$} & \multirow{2}{*}{$\begin{array}{l}\text { Leaf } \\
\text { area } \\
\left(\mathrm{cm}^{2}\right)\end{array}$} & \multicolumn{2}{|c|}{ Leaf colour } & \multirow{2}{*}{$\begin{array}{l}\text { Leaf } \\
\text { shape }\end{array}$} & \multirow[t]{2}{*}{ LM } & \multirow[t]{2}{*}{$\mathrm{PL}$} & \multirow[t]{2}{*}{ LS } & \multirow[t]{2}{*}{$P / A G$} & \multirow{2}{*}{$\begin{array}{l}\text { Time of } \\
\text { leaf fall }\end{array}$} \\
\hline & & & & & $\begin{array}{c}\text { Emerging } \\
\text { leaves }\end{array}$ & $\begin{array}{l}\text { Mature } \\
\text { leaves }\end{array}$ & & & & & & \\
\hline $\begin{array}{l}\text { Shan-i- } \\
\text { Punjab }\end{array}$ & $\begin{array}{l}25^{\text {th }} \\
\text { January }\end{array}$ & 12.33 & 3.36 & 54.83 & $\begin{array}{l}\text { Yellow green } \\
\text { group } 152 \mathrm{~A}\end{array}$ & $\begin{array}{c}\text { Green group } \\
137 \mathrm{~A}\end{array}$ & Lanceolate & SS & 0.80 & $P$ & $\begin{array}{l}\text { Pres- } \\
\text { ent }\end{array}$ & $\begin{array}{l}2^{\text {nd }} \text { De- } \\
\text { cember }\end{array}$ \\
\hline Kanto-5 & $\begin{array}{l}4^{\text {th }} \\
\text { March }\end{array}$ & 18.46 & 4.46 & 154.63 & $\begin{array}{l}\text { Yellow green } \\
\text { group } 152 \mathrm{~A}\end{array}$ & $\begin{array}{c}\text { Green group } \\
136 \mathrm{~A}\end{array}$ & Lanceolate & SS & 1.13 & $P$ & $\begin{array}{l}\text { Pres- } \\
\text { ent }\end{array}$ & $\begin{array}{l}20^{\text {th }} \text { No- } \\
\text { vember }\end{array}$ \\
\hline Fertilia & $\begin{array}{l}11^{\text {th }} \\
\text { March }\end{array}$ & 11.03 & 2.86 & 104.40 & $\begin{array}{l}\text { Yellow green } \\
\text { group } 152 \mathrm{~A}\end{array}$ & $\begin{array}{c}\text { Green group } \\
139 \mathrm{~A}\end{array}$ & Lanceolate & SS & 0.70 & $P$ & $\begin{array}{l}\text { Pres- } \\
\text { ent }\end{array}$ & $\begin{array}{l}4^{\text {th }} \text { De- } \\
\text { cember }\end{array}$ \\
\hline $\begin{array}{l}\text { Yum } \\
\text { Yong }\end{array}$ & $\begin{array}{l}3^{\text {rd }} \\
\text { March }\end{array}$ & 16.66 & 3.95 & 144.40 & $\begin{array}{l}\text { Yellow green } \\
\text { group } 152 \text { B }\end{array}$ & $\begin{array}{c}\text { Yellow } \\
\text { green group } \\
146 \mathrm{~A}\end{array}$ & Lanceolate & SS & 0.77 & $\mathrm{P}$ & $\begin{array}{l}\text { Pres- } \\
\text { ent }\end{array}$ & $\begin{array}{l}18^{\text {th }} \text { No- } \\
\text { vember }\end{array}$ \\
\hline $\begin{array}{l}\text { Florda- } \\
\text { sun }\end{array}$ & $\begin{array}{l}27^{\text {th }} \\
\text { January }\end{array}$ & 13.10 & 4.25 & 109.26 & $\begin{array}{l}\text { Yellow green } \\
\text { group } 152 \mathrm{~A}\end{array}$ & $\begin{array}{c}\text { Green group } \\
133 \mathrm{~A}\end{array}$ & Lanceolate & SS & 0.77 & $\mathrm{P}$ & $\begin{array}{l}\text { Pres- } \\
\text { ent }\end{array}$ & $\begin{array}{l}3^{\text {rd }} \text { De- } \\
\text { cember }\end{array}$ \\
\hline $\begin{array}{l}\text { Sum- } \\
\text { merglo }\end{array}$ & $\begin{array}{l}10^{\text {th }} \\
\text { March }\end{array}$ & 12.70 & 3.56 & 102.03 & $\begin{array}{l}\text { Yellow green } \\
\text { group } 152 \mathrm{~A}\end{array}$ & $\begin{array}{c}\text { Green group } \\
137 \mathrm{~A}\end{array}$ & Lanceolate & SS & 0.53 & $P$ & $\begin{array}{l}\text { Pres- } \\
\text { ent }\end{array}$ & $\begin{array}{l}16^{\text {th }} \mathrm{No}- \\
\text { vember }\end{array}$ \\
\hline $\begin{array}{l}\text { Sun- } \\
\text { coast }\end{array}$ & $\begin{array}{l}2^{\text {nd }} \\
\text { March }\end{array}$ & 18.50 & 4.03 & 145.56 & $\begin{array}{l}\text { Yellow green } \\
\text { group } 152 \text { B }\end{array}$ & $\begin{array}{c}\text { Green group } \\
137 \mathrm{~A}\end{array}$ & Lanceolate & SS & 1.10 & $P$ & $\begin{array}{l}\text { Pres- } \\
\text { ent }\end{array}$ & $\begin{array}{l}12^{\text {th }} \mathrm{No}- \\
\text { vember }\end{array}$ \\
\hline
\end{tabular}

LM: Leaf margin; PL: Petiole length (cm); LS: Leaf surface; P/AG: Presence/absence of glands; SS: Shallow Serrate; P: Pubescent

Table 2: Continue... 


\begin{tabular}{|c|c|c|c|c|c|c|c|c|c|c|c|c|}
\hline \multirow{2}{*}{$\begin{array}{l}\text { Acces- } \\
\text { sion }\end{array}$} & \multirow{2}{*}{$\begin{array}{l}\text { Time of } \\
\text { leaf bud } \\
\text { burst }\end{array}$} & \multirow{2}{*}{$\begin{array}{l}\text { Leaf } \\
\text { length } \\
(\mathrm{cm})\end{array}$} & \multirow{2}{*}{$\begin{array}{l}\text { Leaf } \\
\text { width } \\
(\mathrm{cm})\end{array}$} & \multirow{2}{*}{$\begin{array}{l}\text { Leaf } \\
\text { area } \\
\left(\mathrm{cm}^{2}\right)\end{array}$} & \multicolumn{2}{|c|}{ Leaf colour } & \multirow{2}{*}{$\begin{array}{l}\text { Leaf } \\
\text { shape }\end{array}$} & \multirow[t]{2}{*}{ LM } & \multirow[t]{2}{*}{$\mathrm{PL}$} & \multirow[t]{2}{*}{ LS } & \multirow[t]{2}{*}{ P/AG } & \multirow{2}{*}{$\begin{array}{l}\text { Time of } \\
\text { leaf fall }\end{array}$} \\
\hline & & & & & $\begin{array}{c}\text { Emerging } \\
\text { leaves }\end{array}$ & $\begin{array}{l}\text { Mature } \\
\text { leaves }\end{array}$ & & & & & & \\
\hline $\begin{array}{l}\text { Florda- } \\
\text { belle }\end{array}$ & $\begin{array}{l}18^{\text {th }} \\
\text { January }\end{array}$ & 3 & 3.56 & 6.16 & $\begin{array}{l}\text { Yellow green } \\
\text { Group } 152 \text { B }\end{array}$ & $\begin{array}{c}\text { Green group } \\
133 \mathrm{~A}\end{array}$ & $\begin{array}{l}\text { Lan- } \\
\text { ceolate }\end{array}$ & SS & 0.60 & $P$ & Present & \\
\hline Ambri & $\begin{array}{l}4^{\text {th }} \\
\text { March }\end{array}$ & .90 & 3.70 & 4.70 & $\begin{array}{l}\text { Yellow green } \\
\text { Group } 152 \text { B }\end{array}$ & & $\begin{array}{l}\text { Lan- } \\
\text { ceolate }\end{array}$ & SS & 1.20 & $P$ & Pre & \\
\hline $\begin{array}{l}\text { Early } \\
\text { redhaven }\end{array}$ & $\begin{array}{l}3^{\text {rd }} \\
\text { March }\end{array}$ & 15.03 & 3.33 & 104.58 & $\begin{array}{l}\text { Yellow green } \\
\text { group } 152 \mathrm{~B}\end{array}$ & $\begin{array}{l}\text { Yellow green } \\
\text { group } 137 \mathrm{~A}\end{array}$ & $\begin{array}{l}\text { Lan- } \\
\text { ceolate }\end{array}$ & SS & 0.83 & $\mathrm{P}$ & & \\
\hline Okubo & $\begin{array}{l}8^{\text {th }} \\
\text { March }\end{array}$ & 19.23 & 5.03 & 193.43 & $\begin{array}{l}\text { Yellow green } \\
\text { group } 152 \mathrm{~A}\end{array}$ & $\begin{array}{l}\text { Yellow green } \\
\text { group } 133 \mathrm{~A}\end{array}$ & $\begin{array}{l}\text { Lan- } \\
\text { ceolate }\end{array}$ & SS & 0.73 & $\mathrm{P}$ & Pre & \\
\hline $\begin{array}{l}\text { Early am- } \\
\text { ber }\end{array}$ & $\begin{array}{l}24^{\text {th }} \text { Feb- } \\
\text { ruary }\end{array}$ & 15.16 & 4.16 & 90.66 & $\begin{array}{l}\text { Yellow green } \\
\text { group } 152 \mathrm{~B}\end{array}$ & $\begin{array}{l}\text { Yellow green } \\
\text { group } 136 \mathrm{~A}\end{array}$ & $\begin{array}{l}\text { Lan- } \\
\text { ceolate }\end{array}$ & SS & 0.90 & $\mathrm{P}$ & Present & $\begin{array}{l}23^{\text {rd }} \text { No- } \\
\text { vember }\end{array}$ \\
\hline $\begin{array}{l}\text { Fire } \\
\text { prince }\end{array}$ & $\begin{array}{l}5^{\text {th }} \\
\text { March }\end{array}$ & 16.33 & 3.66 & 149.20 & $\begin{array}{l}\text { Yellow green } \\
\text { group } 152 \mathrm{~A}\end{array}$ & $\begin{array}{l}\text { Yellow green } \\
\text { group } 139 \mathrm{~A}\end{array}$ & $\begin{array}{l}\text { Lan- } \\
\text { ceolate }\end{array}$ & SS & 0.80 & $P$ & Present & $\begin{array}{l}3^{\text {rd }} \mathrm{De}- \\
\text { cember }\end{array}$ \\
\hline $\begin{array}{l}\text { Belle of } \\
\text { georgia }\end{array}$ & $\begin{array}{l}3^{\text {rd }} \\
\text { March }\end{array}$ & 0 & 3.76 & & $\begin{array}{l}\text { Yellow green } \\
\text { group } 152 \mathrm{~A}\end{array}$ & $\begin{array}{l}\text { Yellow green } \\
\text { group } 137 \mathrm{~A}\end{array}$ & $\begin{array}{l}\text { Lan- } \\
\text { ceolate }\end{array}$ & SS & 0.70 & $P$ & Present & $\begin{array}{l}5^{\text {th }} \mathrm{De}- \\
\text { cember }\end{array}$ \\
\hline $\begin{array}{l}\text { Hale ha- } \\
\text { ven }\end{array}$ & $\begin{array}{l}6^{\text {th }} \\
\text { March }\end{array}$ & 14.75 & 3.43 & .23 & $\begin{array}{l}\text { Yellow green } \\
\text { group } 152 \mathrm{~B}\end{array}$ & $\begin{array}{l}\text { Yellow green } \\
\text { group } 135 \mathrm{~A}\end{array}$ & $\begin{array}{l}\text { Lan- } \\
\text { ceolate }\end{array}$ & SS & 0.40 & $\mathrm{P}$ & Present & $\begin{array}{l}10^{\text {th }} \mathrm{De}- \\
\text { cember }\end{array}$ \\
\hline Nishiki & $\begin{array}{l}4^{\text {th }} \\
\text { March }\end{array}$ & 17.46 & 5.03 & 165.40 & $\begin{array}{l}\text { Yellow green } \\
\text { group } 152 \text { B }\end{array}$ & $\begin{array}{l}\text { Yellow green } \\
\text { group } 135 \mathrm{~A}\end{array}$ & $\begin{array}{l}\text { Lan- } \\
\text { ceolate }\end{array}$ & SS & 0.83 & $\mathrm{P}$ & Present & $\begin{array}{l}25^{\text {th }} \text { No- } \\
\text { vember }\end{array}$ \\
\hline Mean & & 15.33 & 3.91 & 124.01 & & & & & 0.80 & & & \\
\hline $\mathrm{CD}(p=0.05$ & & 2.17 & 0.63 & 1.84 & & & & & 0.29 & & & \\
\hline
\end{tabular}

LM: Leaf margin; PL: Petiole length (cm); LS: Leaf surface; P/AG: Presence/absence of glands; SS: Shallow Serrate; P: Pubescent

\begin{tabular}{|c|c|c|c|c|}
\hline Accession & $\begin{array}{l}\text { Time of opening of } \\
\text { first flower }\end{array}$ & Date of full bloom & $\begin{array}{c}\text { Date of opening of last } \\
\text { flower }\end{array}$ & $\begin{array}{c}\text { Duration of } \\
\text { Flowering (Days) }\end{array}$ \\
\hline Shan-i-Punjab & $27^{\text {th }}$ January & $5^{\text {th }}$ February & $20^{\text {th }}$ February & 24 \\
\hline Kanto-5 & $6^{\text {th }}$ March & $11^{\text {th }}$ March & $26^{\text {th }}$ March & 20 \\
\hline Fertilia & $14^{\text {th }}$ March & $20^{\text {th }}$ March & $5^{\text {th }}$ April & 21 \\
\hline Yum Yong & $4^{\text {th }}$ March & $10^{\text {th }}$ March & $24^{\text {th }}$ March & 20 \\
\hline Flordasun & $29^{\text {th }}$ January & $5^{\text {th }}$ February & $25^{\text {th }}$ February & 27 \\
\hline Summerglo & $11^{\text {th }}$ March & $17^{\text {th }}$ March & $29^{\text {th }}$ March & 18 \\
\hline Suncoast & $3^{\text {rd }}$ March & $10^{\text {th }}$ March & $22^{\text {th }}$ March & 20 \\
\hline Florda belle & $20^{\text {th }}$ January & $29^{\text {th }}$ January & $10^{\text {th }}$ February & 21 \\
\hline Ambri & $6^{\text {th }}$ March & $15^{\text {th }}$ March & $26^{\text {th }}$ March & 20 \\
\hline Early redhaven & $5^{\text {th }}$ March & $12^{\text {th }}$ March & $27^{\text {th }}$ March & 22 \\
\hline Okubo & $10^{\text {th }}$ March & $16^{\text {th }}$ March & $31^{\text {th }}$ March & 21 \\
\hline Early amber & $25^{\text {th }}$ February & $4^{\text {th }}$ March & $20^{\text {th }}$ March & 23 \\
\hline Fire prince & $7^{\text {th }}$ March & $18^{\text {th }}$ March & $26^{\text {th }}$ March & 19 \\
\hline Belle of georgia & $5^{\text {th }}$ March & $11^{\text {th }}$ March & $26^{\text {th }}$ March & 21 \\
\hline Hale haven & $8^{\text {th }}$ March & $20^{\text {th }}$ March & $29^{\text {th }}$ March & 22 \\
\hline Nishiki & $2^{\text {nd }}$ March & $11^{\text {th }}$ March & $22^{\text {nd }}$ March & 21 \\
\hline
\end{tabular}


(27 days) and the shortest in Summerglo (18 days). The similar type of variation in duration of flowering was reported by Sharma and Verma (2014), Kumar (2015) and Joshi et al. (2017).

\subsection{Pollen studies}

\subsubsection{Pollen viability}

Pollen viability ranged from $91.35 \%$ in Suncoast to $98.47 \%$ in Belle of Georgia when tested by $2 \%$ acetocarmine and $58.68 \%$ in Kanto- 5 to $93.90 \%$ in FlordaBelle by $0.1 \%$ erythrosin B (Table 4).

\subsubsection{In vitro pollen germination}

\begin{tabular}{|c|c|c|c|c|}
\hline \multirow[t]{2}{*}{ Accession } & \multicolumn{2}{|c|}{ Pollen viability (\%) } & \multicolumn{2}{|c|}{ Pollen germination (\%) } \\
\hline & Acetocarmine $(2 \%)^{*}$ & Erythrosin B $(0.1 \%)^{* *}$ & Sucrose $(10 \%)^{* *}$ & Sucrose $(15 \%)^{* *}$ \\
\hline Shan-i-Punjab & $92.80(9.68)$ & $82.26(65.07)$ & 31.80 (34.24) & 63.60 (52.94) \\
\hline Kanto-5 & $93.33(9.71)$ & $58.68(50.07)$ & $67.31(55.32)$ & 57.34 (49.28) \\
\hline Fertilia & 98.00 (9.95) & $62.97(52.58)$ & $20.26(26.18)$ & $15.59(23.11)$ \\
\hline Yum Yong & $92.33(9.66)$ & 70.15 (57.01) & $11.54(19.85)$ & $42.93(40.91)$ \\
\hline Flordasun & $92.81(9.68)$ & $87.99(69.73)$ & $84.33(66.71)$ & $66.24(55.67)$ \\
\hline Summerglo & $94.48(9.77)$ & $69.70(56.77)$ & $60.46(51.01)$ & $38.84(38.49)$ \\
\hline Suncoast & $91.35(9.60)$ & $61.41(51.63)$ & $19.82(26.41)$ & 46.37 (42.88) \\
\hline Florda belle & $96.39(9.86)$ & $93.90(76.14)$ & $64.98(53.71)$ & 74.22 (60.17) \\
\hline Ambri & $96.17(9.85)$ & $75.61(60.39)$ & 18.53 (25.09) & $11.56(19.83)$ \\
\hline Early redhaven & 93.77 (9.73) & $76.33(60.86)$ & $57.20(49.12)$ & 47.54 (43.57) \\
\hline Okubo & $97.18(9.90)$ & $61.29(51.67)$ & $55.60(48.23)$ & $15.00(22.58)$ \\
\hline Early amber & $97.94(9.94)$ & $68.87(56.11)$ & 26.78 (31.12) & 34.09 (35.45) \\
\hline Fire prince & 97.89 (9.94) & $69.11(56.75)$ & 27.68 (31.73) & $26.45(30.93)$ \\
\hline Belle of georgia & 98.47 (9.97) & $86.32(68.28)$ & 25.19 (29.92) & $22.64(28.21)$ \\
\hline Hale Haven & $93.75(9.73)$ & $75.08(68.03)$ & $17.23(24.50)$ & 41.52 (39.44) \\
\hline Nishiki & $95.16(9.80)$ & $76.00(60.66)$ & 39.83 (39.09) & 54.52 (47.64) \\
\hline Mean & 95.12 & 73.48 & 39.29 & 41.16 \\
\hline $\mathrm{CD}(p=0.05)$ & 0.13 & 8.36 & 6.73 & 11.70 \\
\hline
\end{tabular}

Figure in parenthesis are square root $\left({ }^{*}\right)$ and arc sign $\left({ }^{* *}\right)$ transformed

\section{Conclusion}

From the foregoing discussion, it is concluded that the results obtained are preliminary and there is further need to evaluate these peach accessions in the subsequent years. However, the present studies do indicate that the early flowering varieties have low chilling requirement and can be recommended for cultivation in low hills. and the accessions with high pollen germination can be used as pollinizer in the breeding programme.

\section{References}

Badiyala, S.D., Lakhanpal, S.C., 1994. Performance of some
The in vitro pollen germination in all the peach accessions under study varied from $11.54 \%$ in Yum Yong to $84.33 \%$ in Flordasun under $10 \%$ sucrose solution and $11.56 \%$ in Ambri to $74.22 \%$ in FlordaBelle under $15 \%$ sucrose solution. No definite relationship however, could be established among the staining tests (acetocarmine and erythrosin B) and the pollen germination test. Similar results were observed in different sets of varieties by Josan and Chohan (1980), Kumar et al. (2004), Tandon (2006), Fakim et al. (2011), Yepthomi (2011) and Joshi et al. (2017). The variation in pollen germination percentage so observed may be attributed to the genotypic constitution of the pollen in different accessions.

low chilling peach [Prunus persica (L.) Batsch.] cultivars under Paonta valley conditions of Himachal Pradesh. Himachal Journal of Agricultural Research 20, 57-63.

Bailey, L.H., 1963. The Standard Cyclopedia of Horticulture. McMillan Company, New York. 2423-2439.

Beyers, E., 1962. Diagnostic leaf analysis for deciduous fruits. South African Journal of Agriculutral Sciences 5, 315-29.

Byrne, D.H., Anderson, N., 2012. TexFirst, an early ripening, low chill peach for subtropics. Horticultural Science 47, 1803-04.

Byrne, D.H., 2002. Peach breeding trends. Acta Horticulturae $592,49-59$. 
Chauhan, A., 2016. Characterization of some peach [Prunus persica (L.) Batsch.] germplasm accessions. M.Sc. Thesis. Department of Fruit Science, Dr YS Parmar University of Horticulture and Forestry, Solan. 72.

Fakim, R.S., Hajilou, J., Zaare, N.F., 2011. Pollen germination and pistil performance in several Iranian peach cultivars. International Journal of Agricultural Sciences 4, 170-177.

Government of HP., 2019. Statistical Abstract of Himachal Pradesh. www.admis.hp.nic.in. Accessed in July, 2019.

Hancock, J., Scorza, R., 2008. Peach. In: Hancock, J., (ed). Temperate tree fruit breeding. Springer, New York. 65-298.

Jana, B.R., 2015. Performance of some low chill peach [Prunus persica (L.) Batsch.] under eastern plateau regions of India. International Journal of Current Microbiology and Applied Science 4, 752-57.

Josan, J.S., Chohan, G.S., 1980. Studies on the floral biology of peach under arid-irrigated conditions in Punjab. Progressive Horticulture 12, 47-52.

Joshi, M., Kumar, K., Chauhan, N., Kumari, M., 2017. Selection and Performance of Peach [Prunus persica (L.) Batsch] Cultivars for Hybridization in Himachal Pradesh, India. International Journal of Current Microbiology and Applied Sciences 6, 3714-3722.

Kanwar, J.S., Chanana, Y.R., Kaundal, G.S., 2002. Development of new cultivars of peach for subtropical of India. Acta Horticulturae 592, 103-107.

Kumar, N., Dimri, D.C., Nigam, J.K., 2004. Studies on flowering, fruit set and growth pattern of some promising peach cultivars grown under humid temperate mid hills of Uttarakhand. Indian Journal of Horticulture 61, 271-72.

Kumar, P., 2015. Variability and association studies on low chilling genotypes of peach [Prunus persica (L.) Batsch.].
M.Sc. Thesis. Dr YS Parmar university of Horticulture and forestry, Solan, 69.

Kunden, A., Imrak, B., Rehber, Y., 2004. Peach, Nectarine and plum growing possibilities under subtropical conditions of Turkey and North Cyprus. Acta Horticulturae 662, 119-121.

Meena, B.L., Chandra, A., Kaul, M.K., Meena, R.K., Meena, H.R., 2011. Study on floral biology in peach cultivars in Sriganganagar district of Rajasthan. Progressive Horticulture 42, 315-319.

Sharma, S.K., Verma, K.S., 2014. Adaptation potential of low chill peach varieties to inter-annual climatic variability in the lower Shivalik Himalayas. International Journal of Agriculture, Environment and Biotechnology 7, 157-164.

Singh, A.K., Sharma, R.M., Kher, R., Jasrotia, A., 2005. Introduction and evaluation of pear and peach cultivars under sub-tropics of Jammu region. Acta Horticulturae 696, 25-29.

Tandon, S., 2006. Studies on evaluation of some apricot, peach and plum introductions. M.Sc. Thesis. Dr YS Parmar University of Horticulture and Forestry, Solan, 77.

Wolfe, D., Strang, J., 2010. Peach cultivar performance. University of Kentucky, cooperative Extension Service HO-6. Available from http://www.ca.wky.edu.

Yepthomi, G.I., 2011. Characterization and evaluation of low chilling peach [Prunus persica (L.) Batsch.] germplasm. M.Sc. Thesis. Dr. Y.S. Parmar University of Horticulture and Forestry, Solan, 175.

Yilmaz, K.U., Ercisli, S., Asma, B.M., Dogan, Y., Kafkas, S., 2009. Genetic relatedness in Prunus genus revealed by inter simple sequence repeat markers. Horticultural Science 44, 293-97. 